Hope College

Hope College Digital Commons

Faculty Publications

8-2005

\title{
Extraocular Muscle Activity, Rapid Eye Movements and the Development of Active and Quiet Sleep
}

\author{
Adele M. H. Seelke \\ University of lowa \\ Andrew J. Gall \\ University of lowa, gall@hope.edu \\ Karl ÆE. Karlsson \\ University of lowa \\ Mark S. Blumberg \\ University of lowa
}

Follow this and additional works at: https://digitalcommons.hope.edu/faculty_publications

Part of the Behavioral Neurobiology Commons, and the Biological Psychology Commons

\section{Recommended Citation}

Repository citation: Seelke, Adele M. H.; Gall, Andrew J.; Karlsson, Karl $\mathscr{E}$.; and Blumberg, Mark S., "Extraocular Muscle Activity, Rapid Eye Movements and the Development of Active and Quiet Sleep" (2005). Faculty Publications. Paper 1508.

https://digitalcommons.hope.edu/faculty_publications/1508

Published in: European Journal of Neuroscience, Volume 22, Issue 4, August 1, 2005, pages 911-920.

Copyright @ 2005 Wiley.

This Article is brought to you for free and open access by Hope College Digital Commons. It has been accepted for inclusion in Faculty Publications by an authorized administrator of Hope College Digital Commons. For more information, please contact digitalcommons@hope.edu. 


\title{
EXTRAOCULAR MUSCLE ACTIVITY, RAPID EYE MOVEMENTS, AND THE DEVELOPMENT OF ACTIVE AND QUIET SLEEP
}

\author{
Adele M. H. Seelke, Karl EE. Karlsson, Andrew J. Gall, and Mark S. Blumberg \\ Program in Behavioral and Cognitive Neuroscience Department of Psychology University of lowa, \\ lowa City, IA, 52242, USA
}

\begin{abstract}
Rapid eye movements (REMs), traditionally measured using the electrooculogram (EOG), help to characterize active sleep in adults. In early infancy, however, they are not clearly expressed. Here we measure extraocular muscle activity in infant rats at 3 days of age (P3), P8, and P14-15 in order to assess the ontogeny of REMs and their relationship with other forms of sleep-related phasic activity. We find that the causal relationship between extraocular muscle twitches and REMs strengthens during the first two postnatal weeks, reflecting increased control of the extraocular muscles over eye movements. As early as P3, however, phasic bursts of extraocular muscle twitching occur in synchrony with twitching in other muscle groups, producing waves of phasic activity interspersed with brief periods of quiescence. Surprisingly, the tone of the extraocular muscles, invisible to standard EOG measures, fluctuates in synchrony with the tone of other muscle groups; focal electrical stimulation within the dorsolateral pontine tegmentum, an area that has been shown to contain wake-on neurons in P8 rats, results in the simultaneous activation of high tone in both nuchal and extraocular muscles. Finally, when state-dependent neocortical electroencephalographic activity was observed at P14, it had already integrated fully with sleep and wakefulness as defined using electromyographic criteria alone; this finding is not consistent with the notion that active sleep in infants at this age is "half-activated." All together, these results indicate exquisite temporal organization of sleep soon after birth and highlight the possible functional implications of homologous activational states in striated muscle and neocortex.
\end{abstract}

\section{Keywords}

REM; atonia; myoclonic twitching; electroencephalogram; rat

\begin{abstract}
Sleep and wakefulness are discriminated in adult placental mammals using measures of eye movements, muscle tone, and neocortical activity. This trio of indicators is thought to provide necessary and sufficient information for categorizing sleep-wake states in adults, but its usefulness for categorizing these states in infants is limited (Rechtschaffen \& Kales, 1968). Indeed, although sleep predominates during infancy (Roffwarg et al., 1966; Jouvet-Mounier et al., 1970), the idiosyncratic nature of infant sleep poses unique challenges (Blumberg et al., 2005). For example, before the end of the second postnatal week, infant rats do not exhibit state-dependent electroencephalographic (EEG) activity (Gramsbergen, 1976; Corner \& Mirmiran, 1990; Frank \& Heller, 1997), a feature of infant sleep that has contributed to its characterization as "disorganized," "diffuse," and produced by distinct neurophysiological mechanisms (Adrien \& Lanfumey, 1984; Frank \& Heller, 1997, 2003).
\end{abstract}

Corresponding author: Mark S. Blumberg, Address: E11 Seashore Hall, University of Iowa, Iowa City, IA, 52242, Fax number: (319)-335-0191, e-mail: E-mail: mark-blumberg@uiowa.edu. 
Work from our laboratory has shown that nuchal electromyography (EMG) alone is sufficient for defining sleep-wake states during early infancy (Karlsson \& Blumberg, 2002; Karlsson et al., 2004; Seelke \& Blumberg, 2005). Most recently, it was shown in week-old rats that sleep and wake states identified in this way are governed by neural mechanisms that are similar to those that govern sleep-wake states in adults (Karlsson \& Blumberg, 2005; Karlsson et al., 2005). Thus, of the trio of established indicators of adult sleep-wake states, only the mechanisms underlying rapid eye movements (REMs) have yet to be examined adequately in infants.

Although work in rats, cats, and guinea pigs (Shimizu \& Himwich, 1968; Jouvet-Mounier et al., 1970; McGinty et al., 1977; Van Someren et al., 1990) has addressed the development of REMs using electrooculographic (EOG) techniques, such techniques may not be adequate for assessing the developmental precursors of REMs in very young animals. Thus, although it was reported that rats, in which the eyelids open on postnatal day 15 (P15), first exhibit REMs around P6 (Jouvet-Mounier et al., 1970), it is unclear whether the extraocular muscles that control REMs are active during sleep at earlier ages. If, however, REMs are produced by twitches of the extraocular muscles, as has been suggested (Chase \& Morales, 1983, 1990), and if these extraocular muscle twitches are phenomenologically similar to the twitches produced by other striated muscles, then direct measurement of extraocular muscle activity might reveal ontogenetic precursors of REMs that have heretofore gone unnoticed.

\section{Materials and Methods}

All experiments were performed under National Institutes of Health guidelines for the care of animals in research and were approved by the Institutional Animal Care and Use Committee of the University of Iowa.

\section{Subjects}

A total of 21 P3, P7-9, and P14-15 rats from 21 litters were used. Weights ranged from 8.6$10.1 \mathrm{~g}$ at $\mathrm{P3}, 19.8-20.7 \mathrm{~g}$ at $\mathrm{P} 7-9$, and 37.0-41.1 $\mathrm{g}$ at P14-15. All pups were born to Harlan Sprague-Dawley rats housed in the animal colony at the University of Iowa. The pups were raised in litters that were culled to 8 pups within 3 days of birth (day of birth $=$ Day 0 ). Litters

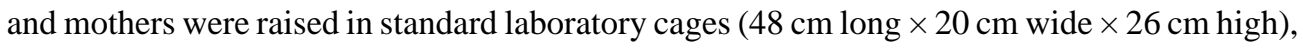
in which food and water were available ad libitum. All rats were maintained on a 12-hr lightdark schedule with lights on at 7 a.m.

\section{Behavioral Recording}

Test Environment-Pups were tested inside an electrically shielded double-walled glass chamber $($ height $=17.0 \mathrm{~cm}$; i.d. $=12.5 \mathrm{~cm}$ ) with a Plexiglas lid. Air temperature inside the chamber was regulated using a temperature-controlled water circulator. Access holes in the side and lid of the chamber allowed for passage of air through the chamber as well as the passage of EMG and EEG electrodes. A round platform constructed of polyethylene mesh was fitted inside the chamber and a perforated felt pad was placed on top of the mesh.

Procedure-Eighteen P3, P8, and P14-15 rats from 18 litters were used. On the day of testing a P3 or P8 rat with a visible milkband was removed from the litter, weighed, and anesthetized with isoflurane. An incision was made in the scalp, which was then retracted to expose the top half of each eyeball. One unipolar stainless steel hook electrode (50 $\mu \mathrm{m}$ diameter, California Fine Wire, Grover Beach, CA) was inserted between the eyeball and orbit on both the nasal and temporal sides of the eye with the aim of implanting in the medial and lateral rectus muscles, respectively. This procedure was repeated for the other eye. The electrodes were secured to the skull with cyanoacrylate adhesive and the scalp was closed. Two bipolar stainless steel 
hook electrodes (California Fine Wire, Grover Beach, CA) were then placed bilaterally in the nuchal muscle and secured with collodion. The subject was lightly restrained on a felt-covered platform, placed in an incubator maintained at thermoneutrality (i.e., $35^{\circ} \mathrm{C}$ ), and allowed to recover for $1 \mathrm{~h}$. (It should be noted that restraint does not interfere with sleep-wake cycling at this age; Karlsson et al., 2004.) The subject was then infused with warm milk (3\% body weight, commercial half-and-half) and allowed to acclimate to the testing chamber (also maintained at $35^{\circ} \mathrm{C}$ ) for $1 \mathrm{~h}$. Testing consisted of collecting $1 \mathrm{~h}$ of both behavioral (using digital video) and electrographic data. After testing the subject was euthanized using an overdose of Nembutal.

A similar protocol was followed for P14-15 subjects. On the day of testing a pup was removed from the litter, weighed, and anesthetized using isoflurane. An incision was made in the scalp, which was then retracted to expose the skull. Holes were drilled over the cerebellum, left parietal, and left frontal cortices, and electrodes, consisting of skull screws (00-96×1/16; Plastics One, Roanoke, VA) attached to insulated silver wires (AG 10T; Medwire, Mt. Vernon, $\mathrm{NY}$ ), were placed in each of the holes to a depth of $1.5 \mathrm{~mm}$. The cerebellar electrode served as the ground. The scalp was then retracted further, exposing the top half of each eyeball, and electrodes were implanted into the extraocular muscles as described above. The scalp was then closed and bipolar stainless steel hook electrodes were implanted bilaterally into the nuchal muscle and secured with collodion. The subject was placed into the testing chamber, maintained at thermoneutrality (i.e., $32^{\circ} \mathrm{C}$ ), for $2-4 \mathrm{~h}$ to recover and acclimate. At this age, pups were tested while freely moving. Electrographic (including extraocular and nuchal EMG and cortical EEG) data were collected for $2 \mathrm{~h}$, including $1 \mathrm{~h}$ in which behavior was recorded to digital videotape. After testing, the subject was euthanized using an overdose of Nembutal.

Nuchal EMG electrodes were connected to differential amplifiers (A-M Systems, Carlsborg, WA) and their signals were amplified $(\times 10,000)$ and filtered $(300-5000 \mathrm{~Hz})$. Extraocular EMG electrodes were filtered from 1-5000 Hz. EEG electrodes were connected to a separate differential amplifier (A-M Systems, Carlsborg, WA) and the signal was amplified $(\times 10,000)$ and filtered $(1-5000 \mathrm{~Hz})$. EMG and EEG data were digitized at $1 \mathrm{kHz}$ using a data acquisition system (BioPac Systems, Santa Barbara, CA) and simultaneously visualized by the experimenter during the test. A microcamera was placed above the chamber lid for monitoring and recording of behavior. EMG and video data were recorded to digital videotape using a data recorder (DV8; WinTron Technologies, Rebersberg, PA).

Data Analysis-Using Spike2 software (CED, Cambridge, UK), extraocular EMG data were filtered from 1-35 Hz to examine gross eye movements (similar to EOG) and from 300-5000 $\mathrm{Hz}$ to examine high-frequency myoclonic twitches.

Peri-event histograms were used to examine the relationship between events in different muscle groups. Two types of comparisons were made. First, we examined the relationship between fluctuations in nuchal and extraocular muscle tone. The nuchal and extraocular EMG data were integrated and full-wave rectified, and the onset of high tone in the nuchal EMG was determined using criteria described previously (Karlsson et al., 2004). In the extraocular EMG trace, the onset of tone in the nuchal muscle was set as the trigger, the 5-s period before and after the trigger was divided into 1-s bins, and the mean amplitude for each bin was determined. Five high-tone onset periods were examined for each subject. The same procedure was repeated for atonia onset. Paired t tests were used to compare adjacent 1-s bins.

Second, we examined the relationship between phasic activity in the extraocular and nuchal muscles. The first twitch of an atonia period in the right extraocular muscle was identified. A twitch was defined as a spike in the EMG that was at least $2 \mathrm{x}$ the noiseband. In the left extraocular muscle trace, the 5-s period before and after the onset of twitching in the EMG of 
the extraocular muscles of the right eye was divided into 1-s bins and the number of twitches in each bin was determined. The same procedure was used when quantifying twitching in the nuchal muscle. Ten atonia periods from each subject were analyzed. Paired t tests were used to compare the amount of twitching in adjacent bins within an age.

The characteristics of rapid eye movements were examined across development. In each subject, twenty eye movements were chosen based upon the following criteria: Eye movements were discrete events exhibiting a deflection from baseline of at least $4 \mathrm{x}$ the noiseband and exhibiting clear onset and offset times. Once an eye movement was identified, its amplitude (defined from peak to trough) and duration were determined and compared across ages using analysis of variance (ANOVA).

The relationship between extraocular muscle twitches and eye movements was also examined. In each subject, twenty extraocular muscle twitches, each preceded by at least $2 \mathrm{~s}$ of quiescence, were selected and the timing of the corresponding eye movement was examined. The number of extraocular muscle twitches that corresponded to the beginning of a rapid eye movement was determined at each age and tested using ANOVA. An identical procedure was used to examine the number of rapid eye movements that were immediately preceded by an extraocular twitch.

A separate analysis examined the development of simultaneous twitching of the left and right extraocular muscles. A twitch in the extraocular muscle of the right eye was selected as a trigger and the extraocular muscle activity of the left eye was examined for evidence of a simultaneous twitch. One hundred twitches were examined for each subject. The percentage of simultaneous twitches was compared across age using ANOVA.

In order to assess limb twitches, 30-min segments of behavioral data from each P3 and P8 pup, and 60-min segments of data from each P14-15 pup, were digitized. As the pup's behavior was observed on a monitor, an event recorder was activated when a myoclonic twitch or highamplitude movement was detected. In this way, a synchronized digital record of the pup's behavior and EMG activity was created. As described elsewhere (Gramsbergen et al., 1970; Blumberg \& Lucas, 1994), twitching was defined as phasic, rapid, and independent movements of the limbs and tail. High-amplitude movements, indicative of wakefulness, include locomotion, stretching, and yawning.

The relationship between multiple limb, nuchal, and extraocular muscle twitches was investigated. Five atonia periods from each $\mathrm{P} 3$ and $\mathrm{P} 8$ subject, and 2 atonia periods from each P14-15 subject, were chosen and divided into 1-s bins. For each subject, data were organized such that each row represented a 1-s bin and each column represented a category of twitching (i.e., limb, extraocular, or nuchal). Each cell was assigned a 1 or a 0 depending on whether a particular category of twitch did or did not occur during that 1-s bin, respectively. Next, in order to determine whether twitching within the three muscle groups was more synchronized than would be expected by chance, the following protocol was used. First, for each subject, the selected atonia periods were divided into quiet sleep (QS) and active sleep (AS) periods, as described previously (Seelke \& Blumberg, 2005) and the QS periods were discarded. Second, the 1s and 0s were summed across columns to produce a PAI for each row of data. Third, observed PAI values were derived for each subject by determining the proportion of rows with PAI values of $0,1,2$, or 3 . Fourth, each of the three columns was shuffled 10 times and the proportion of rows with PAI values of $0,1,2$, or 3 was again calculated; this produced the PAI values expected by chance. Finally, at each age, differences in the observed and expected PAI values were tested using paired $t$ tests.

A spectral analysis of the EEG of P14-15 subjects was conducted using Spike2. AS and QS periods, at least $100 \mathrm{~s}$ in duration and uninterrupted by movement artifact, were chosen for 
analysis; 4 of the 6 P14-15 subjects provided adequate EEG data. One sample of each sleep state was taken from each subject. A Hanning window with 2048 samples was used (bin size $=0.5 \mathrm{~Hz}$ ).

For all tests, alpha was set at 0.05 . Where appropriate, the Bonferroni correction procedure was used. When ANOVA indicated a significant effect, the post hoc test was Fisher's protected least significant difference.

\section{Electrical Stimulation}

Surgery-Three P7-9 rats from 3 litters were used. The pup was prepared for testing using a procedure similar to that described previously (Karlsson et al., 2005). Briefly, to strengthen the skull, it was bleached, dried, and then coated with VetBond (3M, St Paul, MN). Next, a custom built, T-shaped stainless steel head-plant, designed to fit the earbar and nosebar holders of a stereotaxic instrument (David Kopf Instruments, Tujunga, CA), was attached to the skull. Finally, the pup was wrapped in gauze to inhibit movements and induce sleep (Corner \& Kwee, 1976).

Procedure-After recovering for one hour in an incubator maintained at $35^{\circ} \mathrm{C}$, subjects were transferred to a stereotaxic instrument where the pup's body temperature was maintained at $37^{\circ} \mathrm{C}$. Subjects were allowed to acclimate to the stereotaxic instrument for $1 \mathrm{~h}$, at which point they cycle rapidly between high muscle tone and atonia.

Stimulations were performed using concentric, bipolar, tungsten electrodes (1M $\Omega, 3-4 \mu \mathrm{m}$ at the tip, Model TM33CCINS; World Precision Instruments, Sarasota, FL, USA) connected to a stimulus isolator (Model A395; World Precision Instruments, Sarasota, FL, USA) and a stimulus generator (Model 48; Grass, Quincy, MA, USA). As the electrode was lowered into the pons, trains of electrical pulses $(0.2 \mathrm{~ms}, 50-80 \mu \mathrm{A}, 100 \mathrm{~Hz})$ were delivered for $500 \mathrm{~ms}$ every $5 \mathrm{~s}$. When a site was identified that produced high tone in both the extraocular and nuchal muscles, 5 min of EMG data were collected to hard disk. Following data collection, the recording site was marked by passing $50 \mathrm{~mA}$ anodal current through the electrode for $5 \mathrm{~s}$.

Data Analysis-Data were analyzed off-line using Spike2 software. Nuchal and extraocular EMG recordings were rectified and amplitudes were measured over 3 consecutive 500-ms periods: before, during, and after stimulation. Averages from 10 stimulations were calculated for each pup, and paired $t$ tests were performed within each subject comparing mean amplitude during stimulation to the mean amplitude before and after stimulation. For these tests, alpha was set at 0.05 and the Bonferroni correction procedure was used.

\section{Results}

\section{Synchronous fluctuations in extraocular and nuchal muscle tone}

The nuchal EMG fluctuates rapidly in infants between periods of high muscle tone (indicative of wakefulness) and atonia (indicative of sleep) (Karlsson et al., 2004). Surprisingly, during normal cycling between sleep and wakefulness in P3, P8, and P14-P15 rats, the tone of the extraocular muscles fluctuated in lock-step with the tone of the nuchal muscle (Fig. 1A). Specifically, the mean amplitude of extraocular muscle tone increased significantly with the onset of high nuchal muscle tone, and decreased significantly with the onset of nuchal atonia (Fig. 1B). 


\section{Stimulation of the pontine tegmentum increases muscle tone in extraocular and uchal muscles}

It was demonstrated recently that neurons that increase their discharge rates during periods of high nuchal muscle tone are found in the laterodorsal tegmental (LDT) and parabrachial (PB) nuclei (Karlsson et al., 2005); furthermore, lesions of the dorsolateral pontine tegmentum that include these nuclei result in substantial increases in atonia bout durations. Thus, in order to determine whether the near-simultaneous changes in extraocular and nuchal muscle tone are produced by activity within this region, we stimulated the LDT and PB in 3 P7-9 rats. If a common neural mechanism regulates the changes in both extraocular and nuchal muscle tone, then it should be possible to identify sites where stimulation produces simultaneous increases in the tone of both muscle groups.

Electrical stimulation of the PB (Fig. 2A) and LDT (Fig. 2B) produced marked effects on nuchal and extraocular muscle activity. Overall, in all 3 subjects at a total of 4 sites, stimulation of the LDT or PB produced rapid, robust, and significant increases in extraocular and nuchal muscle tone $\left(t_{(9)} \mathrm{S}>5.5, p<0.0005\right)$; muscle tone also decreased significantly after termination of the stimulation at 3 of the 4 stimulation sites $\left(t_{(9)} \mathrm{S}>9.4, p<0.0001\right)$. Stimulation at nearby locations did not produce these effects. These results, in conjunction with the synchronous fluctuations in extraocular and nuchal muscle tone described earlier (Fig. 1), indicate that our method for measuring extraocular EMG provides a valid and reliable measure of extraocular muscle activity.

\section{Developmental relationship between extraocular muscle twitches and REMs}

In addition to indicating changes in tone, nuchal EMG records can reveal the occurrence of myoclonic twitches (Karlsson \& Blumberg, 2002; Seelke \& Blumberg, 2005), as illustrated in the lower trace of Fig. 1A. In light of the hypothesis that REMs represent twitches of the extraocular muscles (Chase \& Morales, 1983), we hypothesized that myoclonic twitches would appear in the extraocular EMG record in infants and would occur in synchrony with REMs at those ages when REMs are detectable.

Examples of extraocular muscle twitches and REMs are depicted in Figure 3A, produced by filtering the extraocular EMG to reveal high- and low-frequency activity, respectively.

Although REMs were easiest to identify at P14-15, at or near the day of eye opening, twitches of the extraocular muscles were apparent as early as P3. As shown in Figure 3B, the duration and amplitude of the eye movements increased significantly with age (duration: $F_{(2,15)}=12.8$, $p<0.001$; amplitude: $\left.F_{(2,15)}=58.5, p<0.0001\right)$.

A tight relationship between extraocular muscle twitches and REMs developed over the first two postnatal weeks. This was demonstrated by calculating the conditional probabilities associated with extraocular muscle twitches and REMs. Specifically, as shown in the left panel of Figure 3C, the occurrence of a twitch increasingly signaled the subsequent occurrence of a REM between P3 and P14-15 $\left(F_{(2,15)}=22.1, p<0.0001\right)$. Conversely, as shown in the right panel of Figure 3C, the occurrence of a REM increasingly signaled the prior occurrence of a twitch between P3 and P14-15 $\left(F_{(2,15)}=74.7, p<0.0001\right)$. Thus, these data indicate that eye movements are gradually brought under the control of the extraocular muscles over the first 2 postnatal weeks.

Age-related changes in the proportion of simultaneous twitches of the extraocular muscles of the 2 eyes were also determined. The percentage of simultaneous twitches increased significantly from $57.5 \pm 7.1 \%$ at $\mathrm{P} 3$ to $74.5 \pm 2.8 \%$ at $\mathrm{P} 8$ to $84.8 \pm 2.9 \%$ at $\mathrm{P} 14-15\left(F_{(2,15)}=\right.$ $8.5, p<0.005)$. Thus, although significant coordination of activity of the extraocular muscles 
of both eyes already exists at P3, this coordination increases significantly over the next 2 weeks and may form the foundation for conjugate eye movements.

\section{Synchronized onset of sleep-related phasic activity in the extraocular and nuchal muscles}

For each atonia period, the first twitch of the extraocular muscles of the right eye was designated as a trigger for a peri-event histogram; then, in relation to this trigger, the number of twitches exhibited by the extraocular muscles of the left eye and the nuchal muscle was determined (Fig. 4). At P3, P8, and P14-15, bouts of twitching were initiated in synchrony in the extraocular muscles of both eyes and the nuchal muscle. These data demonstrate that, during any given atonia period, phasic activity in the form of myoclonic twitching begins synchronously across multiple muscle groups.

\section{Waves of phasic activation during sleep}

If infant sleep is truly "diffuse" and "disorganized," as has been argued (Adrien \& Lanfumey, 1984; Frank \& Heller, 1997, 2003), we would expect twitching in various muscle groups to be randomly distributed throughout each atonia period. We tested this by dividing representative atonia periods into 1-s bins and calculating a phasic activity index (PAI) that reflects, for each bin, the presence or absence of twitching of the limbs, of the extraocular muscles of the right eye, and of the nuchal muscle (see Methods).

Representative atonia periods from P3, P8, and P14 subjects are shown in Fig. 5A. Each atonia period begins with a brief period of quiescence in all 3 categories of twitching $(\mathrm{PAI}=0)$ followed by the onset of phasic activity, which often occurs in synchronized bursts separated by brief periods of quiescence. To determine whether the waxing and waning of phasic activity was more organized than expected by chance, the number of PAIs of 0 and 3 that was experimentally observed was compared with the number of PAIs of 0 and 3 expected by chance (see Methods). At each age tested, the number of observed PAIs of 0 and 3 significantly exceeded the number expected by chance (Fig. 5B; paired $t_{(5)} \mathrm{s}>3.6, p \mathrm{~s}<0.05$ ). Therefore, phasic activity is produced by a mechanism that synchronizes activity across multiple muscle groups.

\section{At P14, state-dependent neocortical activity maps onto EMG-defined sleep-wake tates}

As mentioned above, infant rats do not exhibit state-dependent changes in neocortical EEG until approximately P11 (Gramsbergen, 1976; Frank \& Heller, 1997). Therefore, we next addressed the following question: When slow-wave activity emerges, does it map onto periods of QS defined using measures of nuchal EMG alone?

Fig. 6A shows one representative sleep-wake cycle for a $\mathrm{P} 3$ rat. The cycle begins with a period of high extraocular and nuchal muscle tone (W), followed by a transition to a 6-s period of muscle atonia coupled with behavioral quiescence (QS). Then, a 20-s period of phasic activity commences in the limbs and extraocular and nuchal muscles (AS). After this period of phasic activity, the animal arouses and muscle tone again increases (W).

As shown in Fig. 6B, the basic pattern of sleep-wake activity seen at P3 is also seen at P14, although the duration of each period is elongated. Focusing first on the EMG measures, the cycle begins with a period of high extraocular and nuchal muscle tone (W), followed by a 240s period of muscle atonia coupled with behavioral quiescence (QS). Then, a 250-s period of phasic activity commences in the limbs and extraocular and nuchal muscles (AS). After this period of phasic activity, the animal arouses and muscle tone increases (W). When neocortical EEG activity is considered, it is seen that high-amplitude slow-wave activity overlaps precisely with the period of QS; spectral analysis of this slow-wave activity reveals a frequency peak 
between 2 and $4 \mathrm{~Hz}$, which characterizes delta activity (Fig. 6C, D). Thus, by P14, a tight link between sleep-wake states defined using EEG and EMG criteria has already been established.

\section{Discussion}

The direct measurement of extraocular muscle activity in the present study has revealed features of oculomotor activity during early postnatal life that are invisible to the conventional EOG measure of eye movements. First, and perhaps most surprisingly, fluctuations in the tone of the extraocular muscles mirrored fluctuations in the tone of the nuchal muscle. Second, as early as P3, synchronized waves of phasic activity were seen in the extraocular muscles, nuchal muscle, and limbs; these waves were separated by brief periods of quiescence. Finally, twitches of the extraocular muscles became increasingly associated with robust REMs that resemble those detected in adults using standard EOG techniques. In other words, the extraocular EMG provides a more sensitive measure than the EOG of both tonic and phasic oculomotor activity. The refined organizational structure of infant sleep revealed here contrasts with the perspective of infant sleep as diffuse, primitive, and dissociated (Adrien \& Lanfumey, 1984; Frank \& Heller, 1997, 2003).

The present study depended upon the development of a reliable method for measuring extraocular EMG activity and minimizing movement artifact. This method included a technique whereby the extraocular muscles were approached for implantation of the hook electrode by retracting the scalp and exposing the top half of each eyeball. It was also helpful to secure the elctrodes to the skull near their point of exit from the orbits. In this way, the majority of movement artifact was eliminated. Similar techniques have been used by previous investigators working with infant rats (Jouvet-Mounier et al., 1970; Adrien \& Lanfumey, 1984), although these investigators did not use hook electrodes and filtered their data to reveal only slow-wave activity.

In a recent report (Karlsson et al., 2005), we identified state-dependent neurons within medullary and mesopontine regions of week-old rats that are also considered important for the expression of sleep in adults. For example, neurons were identified within the dorsolateral pontine tegmentum, including the laterodorsal tegmental nucleus (LDT), that increase their discharge rates during periods of elevated nuchal muscle tone. Lesions within this area nearly eradicated the expression of high muscle tone. We extended these results in the present study through electrical stimulation of this region to show that extraocular muscle tone is elevated simultaneously with nuchal muscle tone. Finally, we recently found that carbachol infusion into this region is sufficient to activate high tone in these muscles (Gall \& Blumberg, unpublished data).

We found that at P14-15, neocortical EEG slow-wave activity coincided with the period of QS defined using EMG measures alone (see Figure 6). This observation is interesting in light of the finding that, between P13 and P17, twitches occasionally occur against a background of slow-wave EEG activity (Jouvet-Mounier et al., 1970). Jouvet-Mounier et al. (1970) referred to such episodes as "half-activated paradoxical sleep" or "slow-wave sleep with muscular twitches." These episodes were also reported by McGinty et al. (1977) in their study of sleep in kittens but, as with the study of Jouvet-Mounier et al. (1970), they were relatively infrequent. In their study of sleep development in rats beyond the age of P11, Frank and Heller (1997) also noted the occurrence of half-activated paradoxical sleep and, based largely on this phenomenon, posited their "presleep theory" in which infant sleep is viewed as a single undifferentiated state that is an amalgam of the sleep states seen in adults.

We did not find evidence of a fragmented relationship between EMG and EEG sleep measures at P14. Consider, for example, the events indicated by the arrow in Fig. 6B. First, it can be 
seen that these two events are isolated from the cluster of phasic activity that occurs later during the same atonia period. Second, when we reexamined the video and EMG records, it was apparent that these isolated events were not twitches but, rather, startles. In contrast with twitches, startles are relatively rare, discrete behavioral events characterized by the simultaneous activation of multiple muscle groups (Gramsbergen et al., 1970). We have confirmed the distinction between startles and twitches by monitoring EMG activity simultaneously in nuchal, limb, and abdominal muscles (Viana di Prisco \& Blumberg, unpublished data). Moreover, we have found that startles, but not twitches, are accompanied by hippocampal sharp waves in early infancy (Karlsson \& Blumberg, 2003). It is apparent, then, that startles and twitches are phenomenologically distinct events with distinct patterns of neural activation. Thus, it is possible that the notion of a half-activated sleep state arose from a confusion between startles and twitches. Resolving this issue will require intensive examination of EMG activity and behavior as EEG differentiation occurs around P11. Regardless, it is clear from Fig. 6 that myoclonic twitching in multiple muscle groupsbeginning at P3 - typically occurs in coherent clusters that clearly demarcate periods of active sleep.

Over 20 years ago in a paper examining the spinal mechanisms of twitching in adults cats, Chase and Morales (1983) suggested the possibility that "central neural areas that give rise to myoclonic activation of the limb muscles during REM periods also initiate a generalized pattern of twitches and jerks that affect all striated muscles" (p. 1198), including REMs. The present study, building on our previous report detailing the neural substrates of sleep in week-old rats (Karlsson et al., 2005), provides the most direct experimental support to date for this hypothesis and, moreover, demonstrates its validity soon after birth in an altricial species.

The widely used sleep manual of Rechtschaffen and Kales (Rechtschaffen \& Kales, 1968) established criteria for assessing the stages of sleep that relied on three electrographic measures: EOG, EMG, and EEG (Fig. 7A). Our results, however, indicate that REMs - measured conventionally using the EOG-co-occur with other forms of phasic activity, including the phasic activity that is detectable from the nuchal EMG record. Moreover, extraocular EMG records provide information about muscle tone that is qualitatively and quantitatively similar to that provided by the nuchal EMG. In other words, the extraocular and nuchal muscles control their associated "limbs" - that is, the eyes and head, respectively—and provide redundant information concerning behavioral state. Accordingly, we can restructure the trio of state indicators in Fig. 7A such that, as shown in Fig. 7B, only two measures are now needed to identify behavioral states under normal conditions: EMG and EEG.

But perhaps even two measures is more than is needed. Specifically, it is becoming clear in adult rats that the forebrain exhibits global EEG patterns that are sufficiently distinct to discriminate between AS, QS, and wakefulness (Gervasoni et al., 2004). These authors suggest that these EEG patterns provide the basis for the "classification of global states without reference to behavioral or electromyogram data" (p. 11141). Remarkably, as shown here and elsewhere, the EMG alone is also sufficient for differentiating these same basic sleep-wake states and for revealing neural mechanisms that have been implicated in adult sleep-wake states using the conventional trio of state indicators (Karlsson et al., 2004; Karlsson \& Blumberg, 2005; Seelke \& Blumberg, 2005; Karlsson et al., 2005). Thus, the reduced Venn diagram in Fig. 7B gives way to the qualitatively altered conceptualization of Fig. 7C, in which homologous states defined using either EEG or EMG measures alone are linked by their association with common neural sources within the brainstem, including the mesopontine region.

The perspective captured by Fig. 7C indicates a mechanistic connection between the activational states of the forebrain (EEG) and striated muscle (EMG). Might there also be a 
functional connection? It has been suggested that infant sleep states, including myoclonic twitching, contribute to neural and neuromuscular development (Roffwarg et al., 1966;Mirmiran, 1995;Blumberg \& Lucas, 1996;Corner et al., 2002) and recent reports have provided intriguing evidence in support of this perspective (Petersson et al., 2003;Khazipov et al., 2004). For example, it has been suggested that feedback from twitch-related movements of the limbs contributes to the self-organization of spinal circuits (Petersson et al., 2003). Similarly, it has been suggested that twitches participate in the construction of topographic maps in somatosensory cortex (Khazipov et al., 2004). Both of these reports fit neatly with the perspective being presented here: That the mechanistic link between forebrain and muscle activity derives from a functional linkage that reflects the development of topographic relationships throughout the neuraxis in infants and the modulation and maintenance of these relationships in adults (see Merzenich et al., 1990). Accordingly, this hypothesized link between developmental plasticity and sleep reflects the well-established observation that active sleep predominates during infancy and is retained, albeit at reduced levels, in adulthood (Roffwarg et al., 1966; Jouvet-Mounier et al., 1970).

\section{ACKNOWLEDGEMENTS}

Supported by grants from the National Institute of Mental Health (MH50701, MH66424).

\section{ABBREVIATIONS}

AS, Active sleep; EEG, Electroencephalogram; EMG, Electromyogram; EOG,

Electrooculogram; LDT, Laterodorsal Tegmental nucleus; PAI, Phasic activity index; PB, Parabrachial nucleus; QS, Quiet sleep; REM, Rapid eye movement; W, Wakefulness.

\section{REFERENCES}

Adrien J, Lanfumey L. Neuronal activity of the developing raphe dorsalis: Its relation with the states of vigilance. Experimental Brain Research 1984;8:67-78.

Blumberg MS, Karlsson KÆ, Seelke AMH, Mohns EJ. The ontogeny of mammalian sleep: A response to Frank and Heller (2003). Journal of Sleep Research 2005;14:91-101. [PubMed: 15743339]

Blumberg MS, Lucas DE. Dual mechanisms of twitching during sleep in neonatal rats. Behavioral Neuroscience 1994;108:1196-1202. [PubMed: 7893412]

Blumberg MS, Lucas DE. A developmental and component analysis of active sleep. Developmental Psychobiology 1996;29:1-22. [PubMed: 8719180]

Chase MH, Morales FR. Subthreshold excitatory activity and motoneuron discharge during REM periods of active sleep. Science 1983;221:1195-1198. [PubMed: 6310749]

Chase MH, Morales FR. The atonia and myoclonia of active (REM) sleep. Annual Review of Psychology 1990;41:557-584.

Corner MA, Kwee P. Cyclic EEG and motility patterns during sleep in restrained infant rats. Electroencephalography and Clinical Neurophysiology 1976;41:64-72. [PubMed: 58769]

Corner MA, van Pelt J, Wolters PS, Baker RE, Nuytinck RH. Physiological effects of sustained blockade of excitatory synaptic transmission on spontaneously active developing neuronal networks--an inquiry into the reciprocal linkage between intrinsic biorhythms and neuroplasticity in early ontogeny. Neuroscience and Biobehavioral Reviews 2002;26:127-185. [PubMed: 11856557]

Corner MH, Mirmiran M. Spontaneous neuronal firing patterns in the occipital cortex of developing rats. International Journal of Developmental Neuroscience 1990;8:309-316. [PubMed: 2386080]

Frank MG, Heller HC. Development of REM and slow wave sleep in the rat. American Journal of Physiology 1997;272:R1792-R1799. [PubMed: 9227592]

Frank MG, Heller HC. The ontogeny of mammalian sleep: a reappraisal of alternative hypotheses. Journal of Sleep Research 2003;12:25-34. [PubMed: 12603784] 
Gervasoni D, Lin S, Ribeiro S, Soares ES, Pantoja J, Nicolelis MAL. Global forebrain dynamics predict rat behavioral states and their transitions. The Journal of Neuroscience 2004;24:11137-11147. [PubMed: 15590930]

Gramsbergen A. The development of the EEG in the rat. Developmental Psychobiology 1976;9:501515. [PubMed: 1001836]

Gramsbergen A, Schwartze P, Prechtl HFR. The postnatal development of behavioral states in the rat. Developmental Psychobiology 1970;3:267-280. [PubMed: 5527425]

Jouvet-Mounier D, Astic L, Lacote D. Ontogenesis of the states of sleep in rat, cat, and guinea pig during the first postnatal month. Developmental Psychobiology 1970;2:216-239. [PubMed: 5527153]

Karlsson KÆ, Blumberg MS. The union of the state: Myoclonic twitching is coupled with nuchal muscle atonia in infant rats. Behavioral Neuroscience 2002;116:912-917. [PubMed: 12369810]

Karlsson KÆ, Blumberg MS. Hippocampal theta in the newborn rat is revealed under conditions that promote REM sleep. Journal of Neuroscience 2003;23:1114-1118. [PubMed: 12598598]

Karlsson KÆ, Blumberg MS. Active medullary control of atonia in week-old rats. Neuroscience 2005;130:275-283. [PubMed: 15561443]

Karlsson KÆ, Gall AJ, Mohns EJ, Seelke AMH, Blumberg MS. The neural substrates of infant sleep in rats. PLoS Biology 2005;3:e143. [PubMed: 15826218]

Karlsson KÆ, Kreider JC, Blumberg MS. Hypothalamic contribution to sleep-wake cycle development. Neuroscience 2004;123:575-582. [PubMed: 14698764]

Khazipov R, Sirota A, Leinekugel X, Holmes GL, Ben-Ari Y, Buzsaki G. Early motor activity drives spindle bursts in the developing somatosensory cortex. Nature 2004;432:758-761. [PubMed: 15592414]

McGinty D, Stevenson M, Hoppenbrouwers T, Harper RM, Sterman MB, Hodgman J. Polygraphic studies of kitten development: Sleep state patterns. Developmental Psychobiology 1977;10:455-469. [PubMed: 199512]

Merzenich, MM.; Recanzone, G.; Jenkins, WM.; Nudo, RJ. How the brain functionally rewires itself. In: Arbib, M.; Robinson, J., editors. Natural and Artificial Parallel Computations. Cambridge, MA: MIT Press; 1990. p. 177-210.

Mirmiran M. The function of fetal/neonatal rapid eye movement sleep. Behavioural Brain Research 1995;69:13-22. [PubMed: 7546304]

Petersson P, Waldenström A, Fåhraeus C, Schouenborg J. Spontaneous muscle twitches during sleep guide spinal self-organization. Nature 2003;424:72-75. [PubMed: 12840761]

Rechtschaffen, A.; Kales, A. A manual of standardized terminology, techniques, and scoring system for sleep stages of human subjects. Los Angeles: UCLA Brain Information Service/Brain Research Institute; 1968.

Roffwarg HP, Muzio JN, Dement WC. Ontogenetic development of the human sleep-dream cycle. Science 1966;152:604-619. [PubMed: 17779492]

Seelke AMH, Blumberg MS. Thermal and nutritional modulation of sleep in infant rats. Behavioral Neuroscience 2005;119:603-611. [PubMed: 15839806]

Shimizu A, Himwich H. The ontogeny of sleep in kittens and young rabbits. Electroencephalography and Clinical Neurophysiology 1968;24:307-318. [PubMed: 4174003]

Van Someren EJW, Mirmiran M, Bos NPA, Lamur A, Kumar A, Molenaar PCM. Quantitative analysis of eye movements during REM-sleep in developing rats. Developmental Psychobiology 1990;23:5561. [PubMed: 2340957] 
A

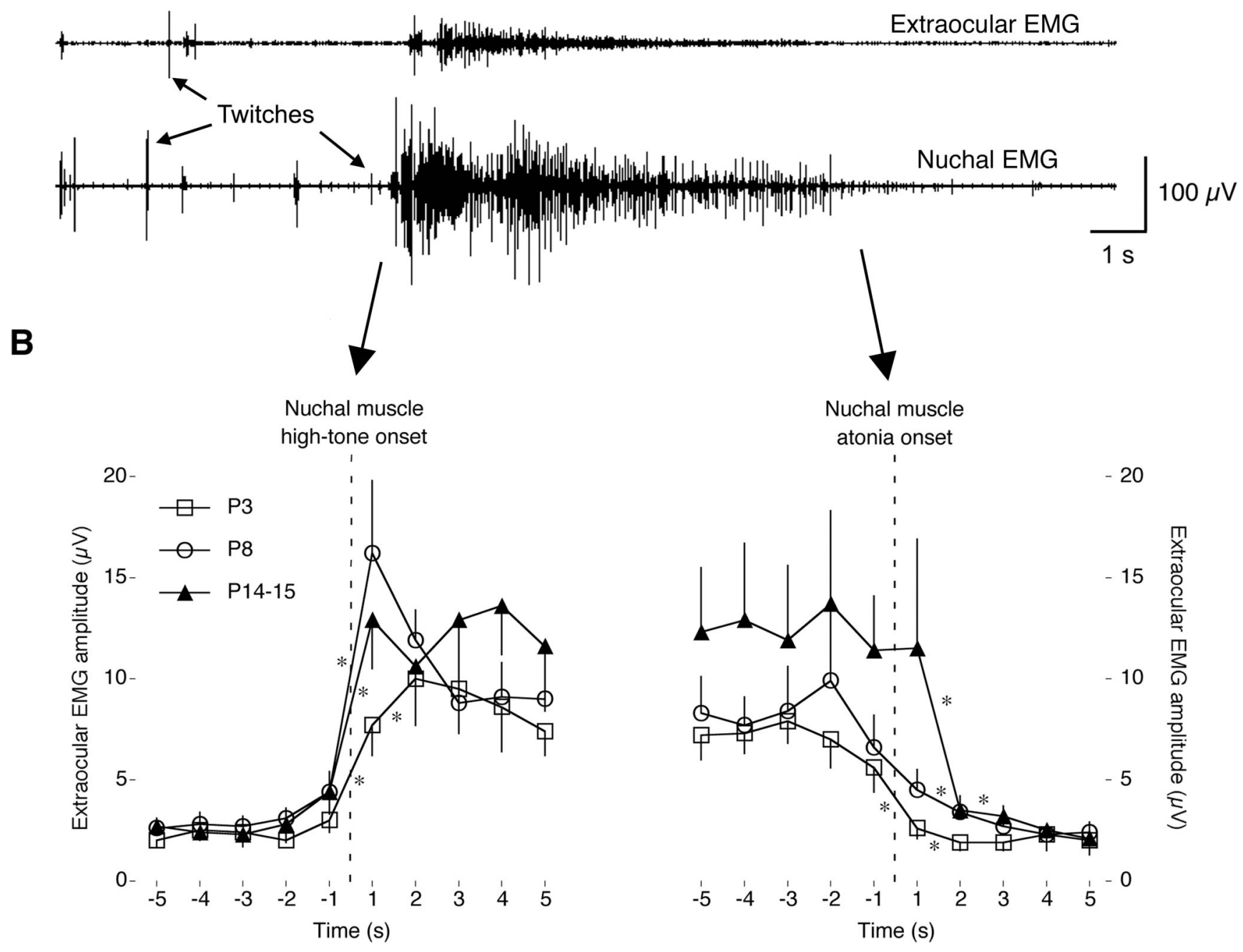

Figure 1.

Extraocular muscle activity fluctuates in synchrony with nuchal muscle activity. (A)

Representative EMG activity for the extraocular (upper trace) and nuchal (lower trace) muscles in a P8 rat. Two periods of atonia (indicative of sleep) separated by a period of high muscle tone (indicative of wakefulness) can be seen. Myoclonic twitches (indicative of active sleep) are also indicated. (B) Mean changes in EMG amplitude for the extraocular muscles in relation to the onset of high nuchal muscle tone (left panel) and the onset of nuchal atonia (right panel) for $\mathrm{P} 3, \mathrm{P} 8$, and $\mathrm{P} 14 / 15$ rats. $\mathrm{n}=6$ pups/group. ${ }^{*} p<0.05$ in relation to the previous time point. Mean \pm s.e. 
A

Nuchal

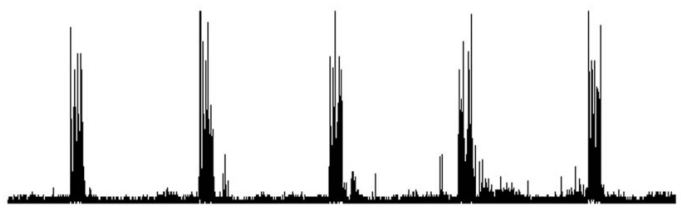

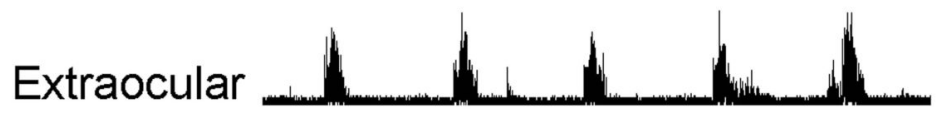

Stimulation

B
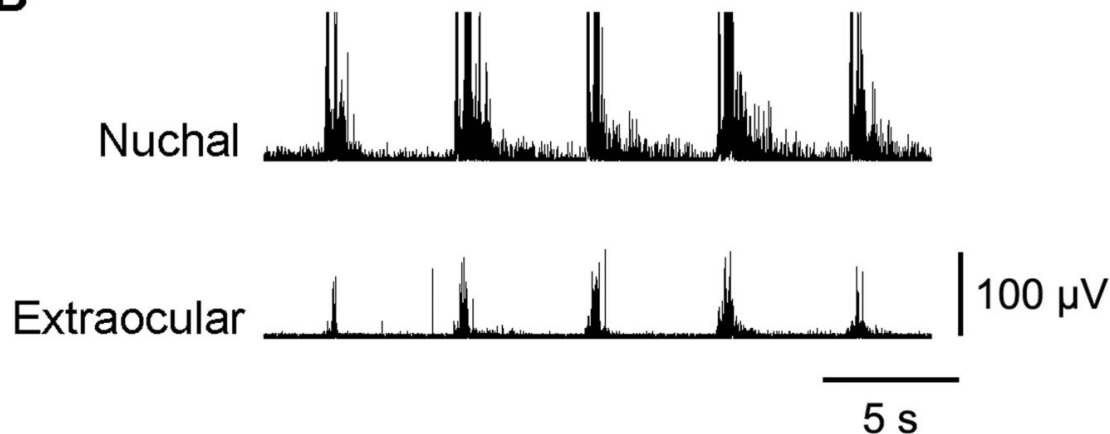

C

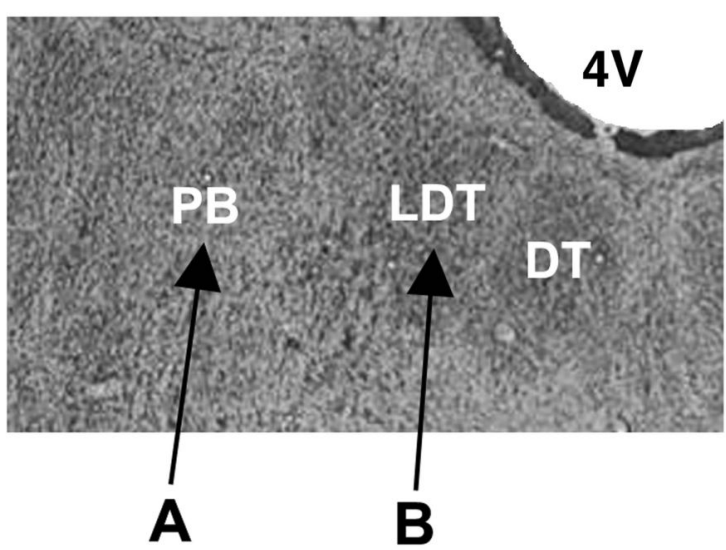

Figure 2.

Stimulation within the dorsolateral pontine tegmentum simultaneously activates high tone in the nuchal and extraocular muscles. (A) Nuchal and extraocular EMG activity in response to stimulus trains of electrical pulses (vertical bars) delivered to the parabrachial nucleus (PB) of a P9 rat. Each pulse was delivered for $500 \mathrm{~ms}(60 \mu \mathrm{A}, 100 \mathrm{~Hz})$. (B) Same as (A) except the stimulation was applied to the laterodorsal tegmental nucleus (LDT) of a P9 rat. (C) Coronal section from an infant rat indicating the locations of the stimulation electrodes in (A) and (B). DT: dorsal tegmental nucleus; $4 \mathrm{~V}: 4^{\text {th }}$ ventricle. 


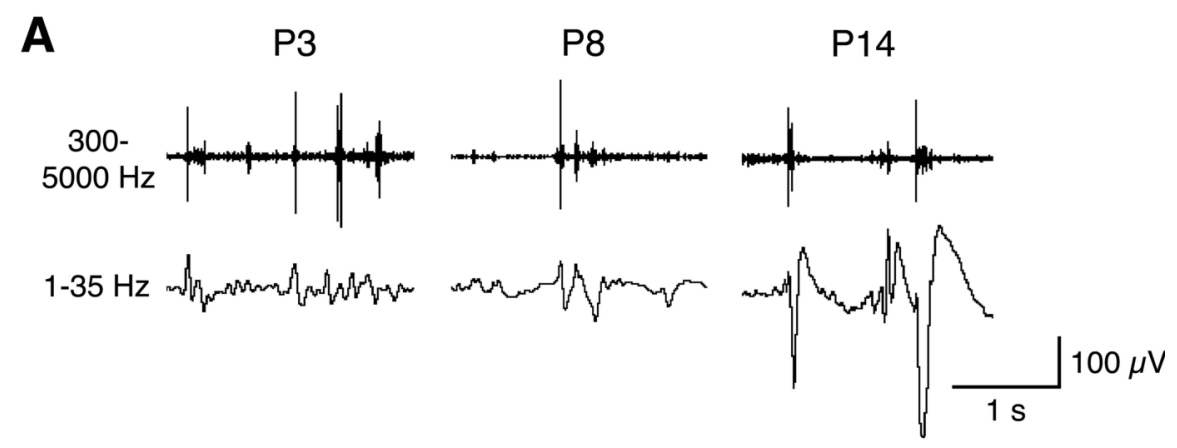

B
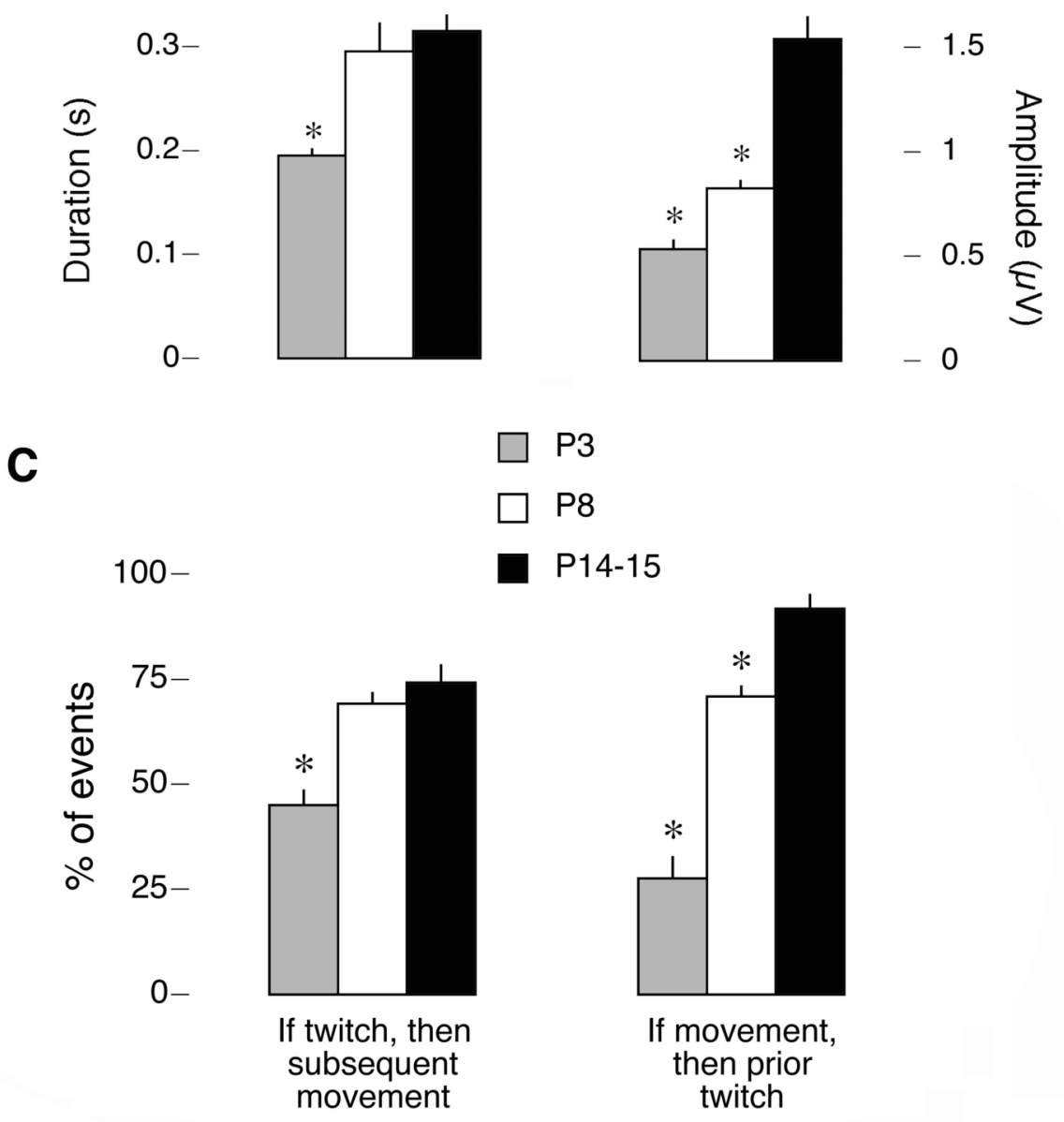

P3

P8

P14-15

Figure 3.

Coupling between extraocular muscle twitches and REMs increases during the first two postnatal weeks. (A) Representative extraocular EMG activity and eye movements at P3, P8, and P14. Top row: High-pass $(300-5000 \mathrm{~Hz})$ filtering to reveal myoclonic twitching. Bottom row: Low-pass (1-35 Hz) filtering to reveal REMs. (B) Analysis of the mean duration (left panel) and amplitude (right panel) of REMs at P3, P8, and P14-15. $\mathrm{n}=6$ pups/group; ${ }^{*} p<$. 001 in relation to the other two groups. (C) Analysis of conditional probabilities relating extraocular muscle twitches to REMs at P3, P8, and P14-15. Left panel: Percentage of events in which an extraocular muscle twitch occurred and was followed by a REM (i.e., REM | twitch). Right panel: Percentage of events in which a REM occurred which was preceded by 
an extraocular muscle twitch (i.e., twitch $\mid$ REM). $\mathrm{n}=6$ pups/group. ${ }^{*} p<0.0005$ in relation to the other two groups. Mean \pm s.e. 


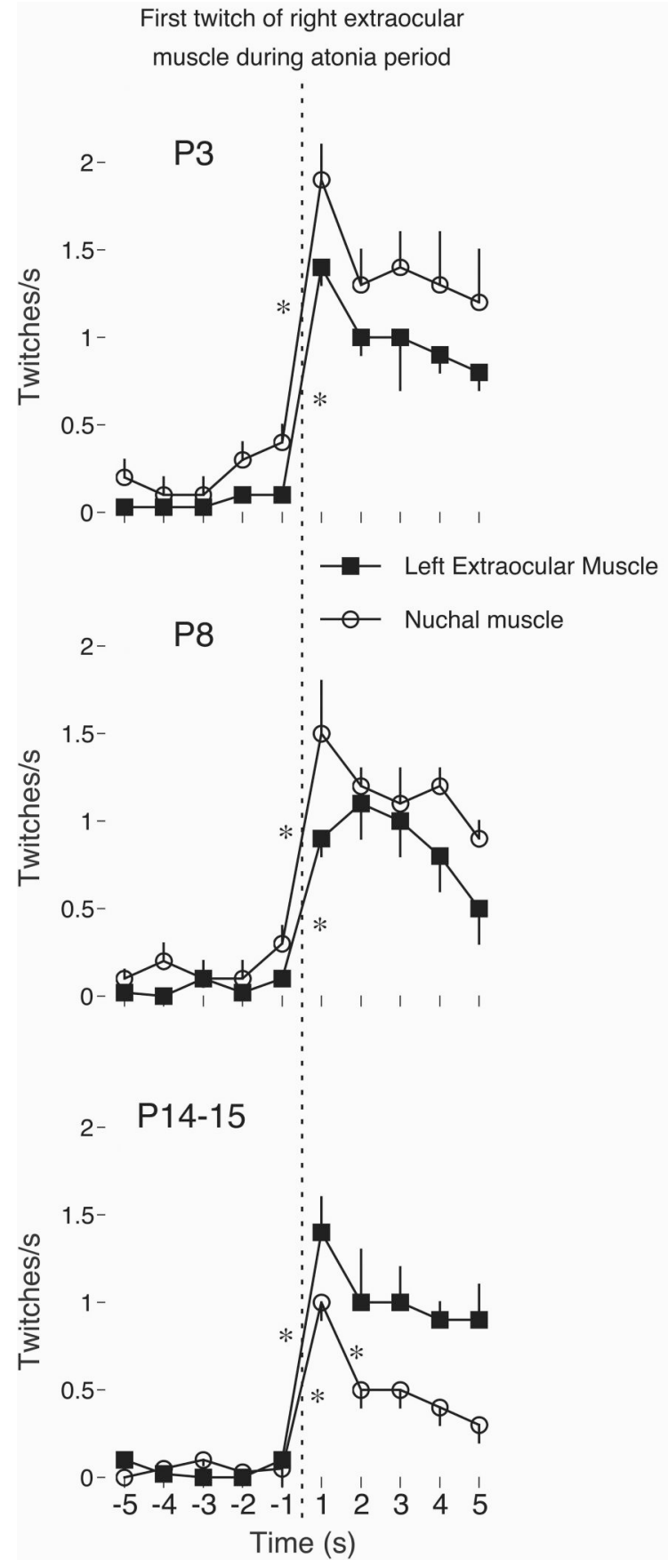

Figure 4.

Synchronized onset of phasic activity in extraocular and nuchal muscles during sleep at P3, P8, and P14-15. The mean number of myoclonic twitches per s determined from the left extraocular and nuchal EMGs in relation to the first twitch of the right extraocular muscle during a period of atonia. $\mathrm{n}=6 \mathrm{pups} / \mathrm{group} ;{ }^{*} p<0.05$ in relation to the previous time point. Twitches were determined from the extraocular and nuchal EMGs. Mean \pm s.e. 
A
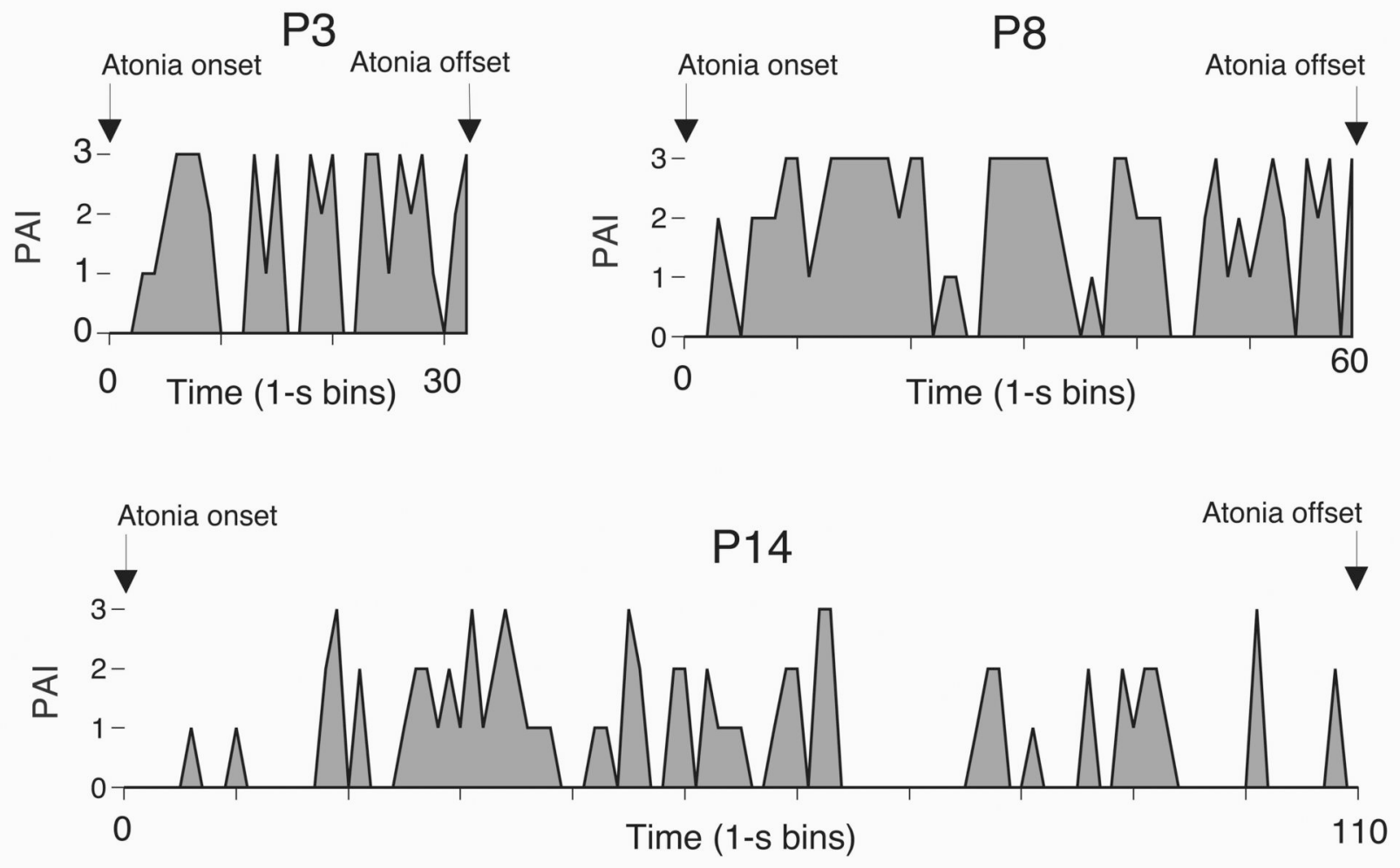

B

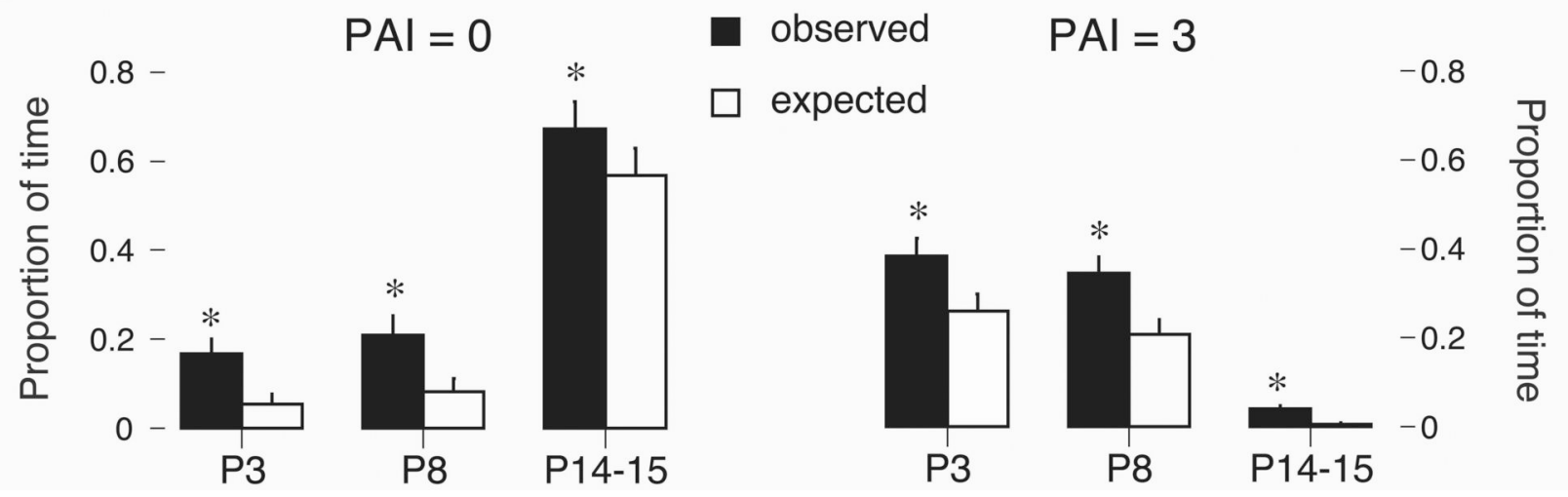

Figure 5.

Synchronized phasic activity in multiple muscle groups during sleep at P3, P8, and P14-15. (A) Representative depictions of phasic activity during atonia periods at each age. The phasic activity index (PAI) was determined for each 1-s bin by assessing myoclonic twitching of the limbs (based on behavioral observation) and of the nuchal and extraocular muscles (based on EMG activity). Four categories were identified ranging from $\mathrm{PAI}=0$ (no phasic activity) to $\mathrm{PAI}=3$ (phasic activity detected in the limbs and nuchal and extraocular muscles). (B) Mean proportion of observed $1-\mathrm{s}$ bins in which $\mathrm{PAI}=0$ (left panel) and $\mathrm{PAI}=3$ (right panel) in relation to the expected values expected by chance. $\mathrm{n}=6 \mathrm{pups} / \mathrm{group} ;{ }^{*} p<0.05$ in relation to the expected value. Mean \pm s.e. 
A

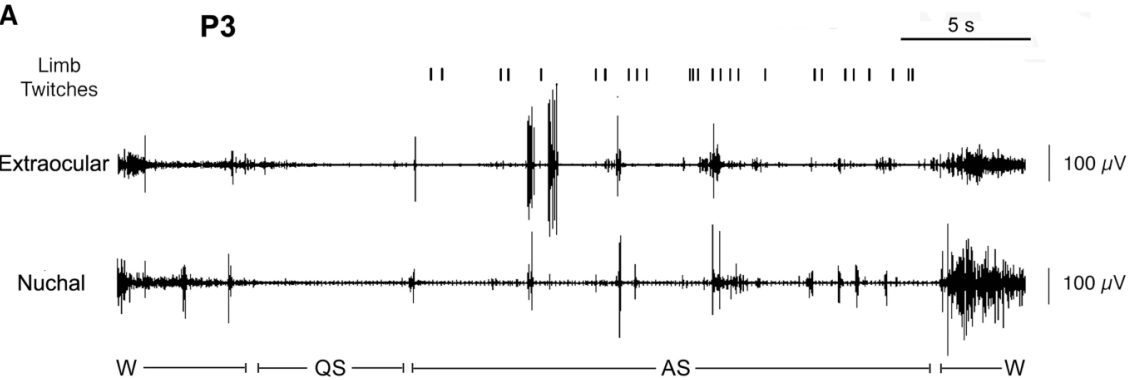

B

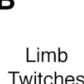

Twitches

P14

Extraocular

Nuchal

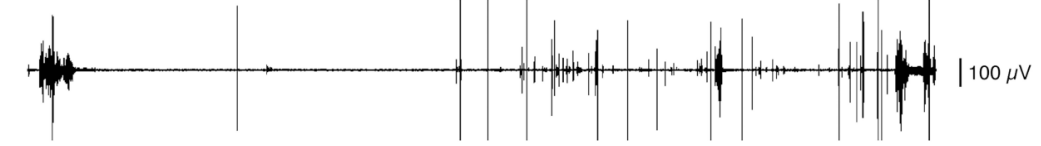

EEG

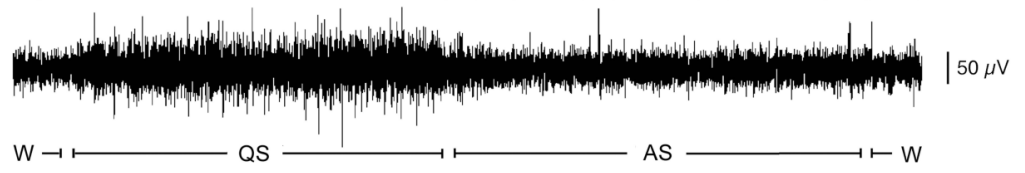

C

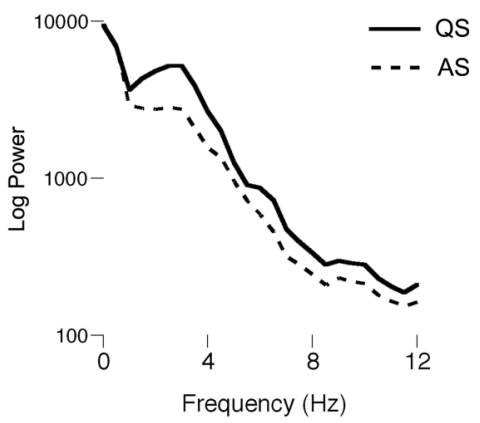

D

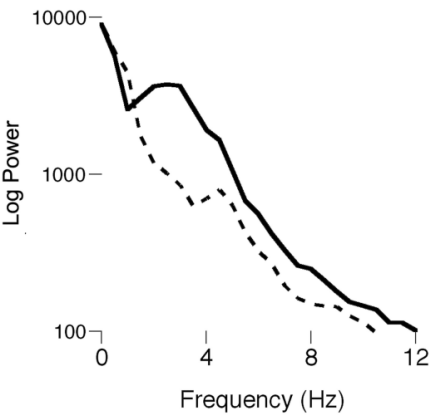

Figure 6.

Phasic and tonic behavioral and electrographic events across a complete sleep cycle in a P3 and P14 rat. (A) Behaviorally scored limb twitches and extraocular and nuchal EMGs for a P3 rat. Periods of wakefulness (W), quiet sleep (QS), and active sleep (AS) are indicated. (B) Same as (A) except the subject is a P14 rat and neocortical EEG is also recorded. Arrow indicates two twitch-like events that were determined to be startles. Note the different time scales for (A) and (B). (C) EEG power spectrogram of the QS and AS periods in (B). (D) EEG power spectrogram of QS and AS periods across four P14-15 subjects (at least $100 \mathrm{~s}$ of continuous data per sleep state per subject). 
A

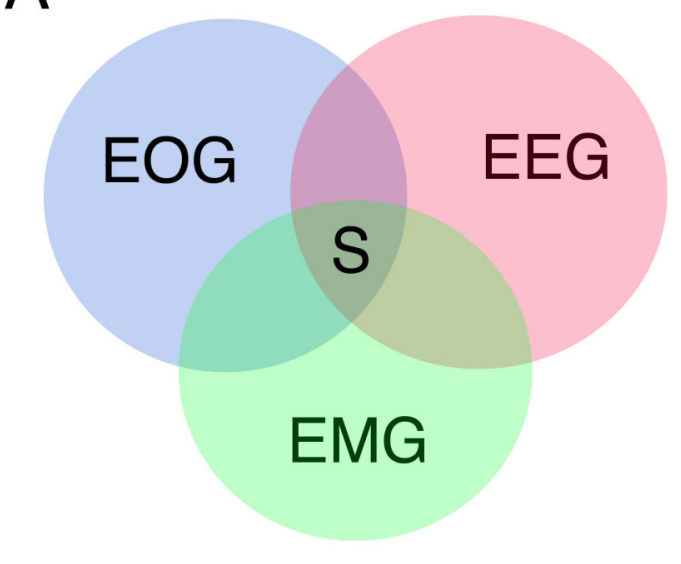

B

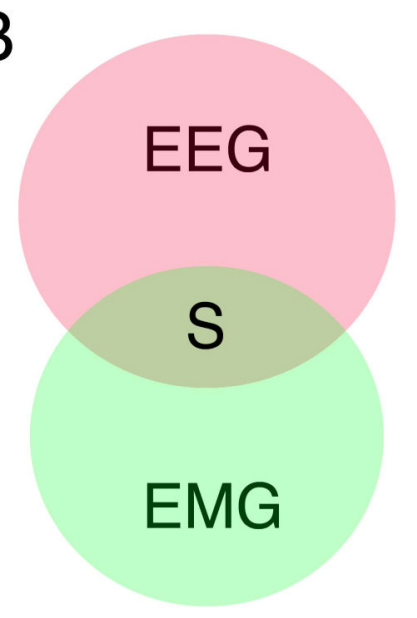

C

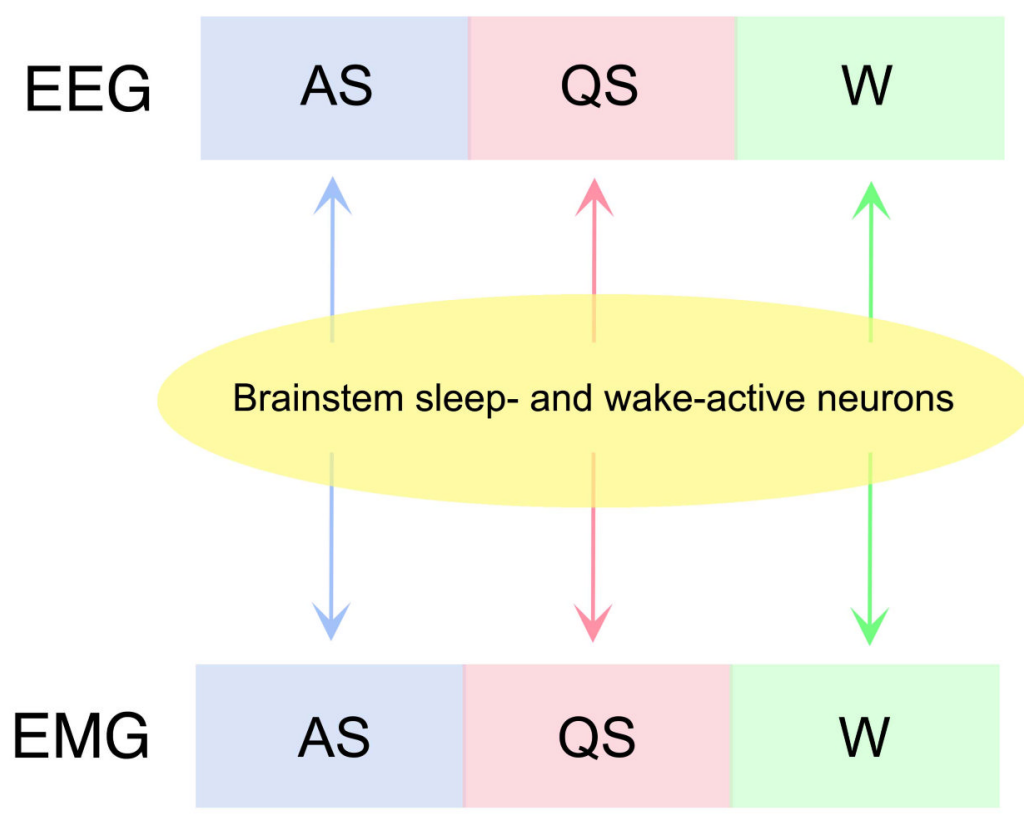

Figure 7.

Conceptual representations of sleep. (A) Venn diagram depicting conventional diagnostic criteria for assessing sleep-wake states in adults using three independent parameters: EOG, nuchal or chin EMG, and neocortical EEG. S denotes the behavioral state (i.e., active or quiet sleep, wakefulness) defined using these three parameters. (B) Reorganization of the Venn diagram in (A) based on the notion that the EOG and EMG provide redundant information. Specifically, REMs can be viewed as phasic events that are analogous to myoclonic twitches that can be recorded using nuchal EMG; in addition, fluctuations in nuchal muscle tone are mirrored by fluctuations in extraocular muscle tone. Now, S denotes the behavioral state defined using only two parameters. (C) Alternative conceptualization that builds on the notion that either EEG (after P11) or EMG (as early as P2) is alone sufficient to define behavioral states in rats. According to this notion, individual homologous sleep-wake states (AS, QS, W) 
can be identified on the basis of either EEG or EMG activity alone. This homology arises because EEG- and EMG-defined states are generated by common brainstem mechanisms. 